\title{
CT Scans in Primary Survey for Polytrauma Patients
}

\author{
Purnajyoti Banerjee $^{1}$, Soumen Rudra ${ }^{1}$, Madhusree Ghosh ${ }^{2 *}$, Praveen Panose ${ }^{1}$ \\ ${ }^{1}$ Department of Trauma \& Orthopaedics, East Surrey Hospital, Redhill, UK \\ ${ }^{2}$ Department of Obstetrics \& Gynaecology, St George’s Hospital, London, UK \\ Email: "banerjee_purnajyoti@yahoo.co.in
}

Received November 30, 2012; revised January 2, 2013; accepted January 10, 2013

Copyright (C) 2013 Purnajyoti Banerjee et al. This is an open access article distributed under the Creative Commons Attribution License, which permits unrestricted use, distribution, and reproduction in any medium, provided the original work is properly cited.

\begin{abstract}
Managing critically injured patients are challenging. Victims of major trauma often present with a host of unknowns including the exact site and nature of injury with a need for urgent diagnosis and resuscitation. It takes significant clinical expertise to detect multiple injuries and implement life or limb saving treatment within a short period of time. Conventional radiographs and focused assessment with sonography for trauma (FAST) scans are useful adjuncts to help in initial diagnosis. Conventional computer tomography (CT) investigations have been used for secondary survey in these scenarios. We reviewed the guidelines that regulate the use of CT scans in multiple injured patients in the UK. CT imaging is rapidly emerging as an adjunct to primary survey. It allows quick detection of major organ injury allowing focussed treatment whilst simultaneous initial resuscitation is underway. Availability of adequate resources is needed for widespread adaptation of this technique that involves manpower, relocation and refurbishment of the CT suites. Although it is not yet clear if using CT imaging during primary survey reduces mortality, it surely results in more organised patient care and efficient use of resources in an acute setting.
\end{abstract}

Keywords: CT; Polytrauma; Primary Survey

\section{Introduction}

Management of patients with multiple injuries is challenging. With improvement in pre hospital care and advancement in early diagnosis of life threatening injuries, the mortality in polytrauma patients have substantially decreased in last thirty years [1]. Major trauma is still one of the leading causes of death amongst young adults in the industrial world. Furthermore, it has a severe socio-economic impact and can result in devastating morbidity especially among the working population [2]. Specific problems associated with initial assessment of polytrauma victims in the emergency department includes patients being confused, unconscious or under the influence of drugs and/or alcohol. It is often very difficult to get an accurate clinical history and life or limb threatening injuries are not apparent in most cases [3]. Accurate interpretations of the available clinical signs and mechanism of injury are the key features to avoid missing potential life threatening injuries that may endanger patient's life. Most emergency departments in the UK work with an agreed round the clock multi-disciplinary trauma team that is available to review any major trauma patients with few minutes of presentation. The

${ }^{*}$ Corresponding author. hospitals are usually informed of these patients by the pre hospital teams attending the patient at the site of accident. This allows adequate time for the team to assemble and resuscitate patients in an organized manner following advanced trauma life support guidelines (ATLS) algorithms [4]. The trauma team consists of anaesthesists, general surgeons, orthopaedic surgeons, radiologists and an emergency department consultant who usually acts as team leader. It is important to follow an agreed guideline as deviations from standard protocols have shown to increase mortality in major trauma patients [5]. The emphasis of managing polytrauma patients are largely based on the "golden hour in shock" principle which calls for focused and effective recognition and treatment of life and limb threatening injuries. Radiological assessment is an essential part of this strategy. The primary radiological survey is undertaken within minutes of patient's arrival in the emergency department [6]. These include an anteroposterior view of the pelvis, a postero-anterior (PA) view of the chest and a single adequate lateral view of the cervical spine including C7. These images can help in early diagnosis of possible life threatening injuries like severe bleeding or unstable fractures that might cause permanent disability in a trauma patient. Focused as- 
sessment with sonography for trauma (FAST) scans are used as an adjunct in the emergency department to help detecting major abdominal or pelvic organ damage. Results of these investigations have a direct impact on the further diagnostic and therapeutic strategy such as transfusion, immediate surgery or urgent transfer to specialist trauma centers. Conventional computer tomography (CT) investigations have been used for secondary survey in the past to assess brain, thoracic, abdominal visceral and pelvic ring lesions in patients that were haemodynamically stable. There are practical limitations of using CT scans in this group like risks of transferring intubated patients from Emergency department to the CT suite as the procedure is time consuming. Furthermore, it was often perceived as a waste of time when such patients warranted emergency surgery [7]. There is evidence that clinical evaluation of polytrauma patients based on conventional history taking, clinical examination and conventional CT scan is often time consuming [8].

With the introduction of spiral computed tomography, and particularly multidetector/multislice CT (MSCT) this scenario has changed considerably. MSCT leads to a reduction of the scanning time due to an increased speed of image acquisition [9-11]. Multiple body parts can be examined with a high resolution and there is the ability to obtain multiplanar reconstructions and immediate onand off-line interpretation of the images at separate workstations. These important aspects contribute to the introduction of the MSCT in the primary survey of severely injured patients. The main advantages of MSCT include a significant reduction in time required to undertake these scans. Some studies indicate, they can be performed within twenty minutes of presentation to the emergency department and the time taken to perform the scan can be as little as six minutes [12]. This is almost $50 \%$ reduction in time taken compared to the conventional CT scan [13]. The main limitation of this technique is availability of a trained radiologist and radiographer round the clock which has cost implications. Furthermore, the CT suite should be adequately equipped so that the patient can be resuscitated simultaneously whilst having the CT scan [14].

The aim of this paper is to review the current guidelines on performance of CT scans in polytrauma patients in the UK. We reviewed the guidelines outlined by advanced trauma life support (ATLS), Royal College of Radiologists (RCR) and British Orthopaedic Association (BOA) on undertaking CT scans in management of multiple injured patients nationally. We have further discussed the role of using CT scan in primary survey of critically injured patients in a polytrauma situation.

\section{History \& Background}

Godfrey Hounsfield (1971) first introduced scanning into medical practice with a successful scan on a patient in Wimbledon. CT scanner turned out to be one of the most revolutionary developments in medical imaging of the century. CT scan technology has advanced rapidly in recent years, moving to more efficient and stable detectors, more refined engineering and data acquisition systems and electronics, and faster computers.

These developments have been largely directed towards faster scanning of further lengths of the patient, using finer slices. As a result, CT scanners have evolved from a slice-by-slice diagnostic imaging system into a truly volumetric imaging modality, where images can be reconstructed in any plane without significant loss of image quality. This has led to the increased use of multiplanar and 3D display modes in diagnosis.

The role of CT imaging in modern management of trauma patients started to evolve with the introduction of contrast enhanced CT scans. This technique can be used to detect major injuries in all regions of the body with reasonable accuracy and using a single imaging modality [15]. The advent of MSCT has revolutionised CT imaging in polytrauma situation. These scans can detect active source of bleeding and major visceral injury with extravasation of the contrast. Furthermore, the information provided by CT scan on admission and subsequently during follow up can enhance patient care even in case where poly trauma patients are treated conservatively [16].

\subsection{Advanced Trauma Life Support Guidelines (ATLS) [4]}

Conventional CT scan of head, chest abdomen and spine is included as an adjunct to secondary survey. Helical contrast CT has been shown to be accurate for blunt aortic injury, CT scan should be undertaken liberally. Patients who are haemodynamically abnormal should not be placed in a CT scanner. A properly performed and interpreted helical CT that is normal may require transfer to higher level of care. ATLS guidelines suggest that CT imaging is a time consuming procedure and should only be done in patients with no haemodynamically abnormalities and where there is no apparent indications of emergency laparotomy. Some Gastrointestinal, diaphragmatic and pancreatic injury may be missed on CT. Evaluation of bladder and pelvis using CT scan is an alternative study that is useful in providing additional information. Suspected urinary system injuries are best evaluated by contrast enhanced CT scan. During evaluation of blunt trauma, if there is early or obvious evidence that the patient will be transferred to another facility, time consuming tests like CT should not be performed.

In penetrating trauma, double or triple contrast CT are useful in flank and back injuries. Contrast enhanced CT scan helps to assess the retroperitoneal colon on the side 
of the wound it has been reported that CT scan is most specific 92\% - 98\% in blunt abdominal trauma. The disadvantages are cost and time for transport.

\subsection{Royal College of Radiology (RCR) Guidelines [17]}

In emergency department, the trauma team leader should decide on the immediate need for CT scan in a polytrauma patient following standard established protocols. Ideally all multiple injured patients should be reviewed by a consultant led team ensuring availability of experienced staff during initial management of these critical patients. All trauma patients should be assessed as soon as possible with minimal movement of the patient in the emergency department to prevent secondary damage to body parts. The CT suite should ideally be located near the emergency department, thereby avoiding long distance patient movement within the hospital. There should be no delay to transfer these patients to the CT suite if the trauma team agrees on an early scan. They should not wait for a conventional radiographs or FAST scan in this situation. It is good practice to ensure that CT scanning in polytrauma comply with the Ionizing Radiation (Medical Exposure) Regulations 2000 [IR (ME) R]. Measures must be taken before transferring the patients to CT suite to enhance quick and safe imaging. These include an intravenous access to ensure contrast injection, a pelvic stabilisation in case of suspected pelvic fracture, adequate splinting of deformed limbs, urinary catheterisation and pregnancy test to exclude untoward radiation of pregnant women. However, the health of the mother will take a preference over the health of the foetus in this scenario.

Whole-body contrast-enhanced MSCT is the default imaging procedure of choice in polytrauma patients. This has been shown to be a predictor of survival compared to conventional CT imaging [18].

There should be a clear protocol and uniform access to this imaging across the regional trauma network to ensure smooth transfer of images to the tertiary level 1 trauma centre where necessary. If there is any sign of major organ injury, the consultant radiologist must immediately inform the trauma team leader. Although, the NHS Clinical Advisory Groups (CAGs) [19] report on regional trauma network suggests patients should undergo CT scan when they are "stable enough". The RCR guidelines suggests that patients should undergo MSCT if they are haemodynamically unstable as the need for the accurate diagnosis to allow immediate focussed surgery/ intervention is greater in these group of patients. It is recognised that small minority of patients will be too unstable to undergo CT at this stage, but most of them will need some surgical intervention in the emergency department itself. Furthermore, the decision to undertake a CT scan as a tool for primary survey lies with the trauma team leader.

The primary survey report is issued immediately to the trauma team leader whilst the final report is usually available within an hour of imaging once it is validated by the on call consultant radiologist. Teleradiology facilities at home for review by the consultant radiologist further hastens this process.

In multiple injured children, similar guidelines have been devised. The salient features include MSCT imaging with rectal contrast in suspected trauma to abdomen or pelvic cavity. In haemodynamically unstable patients CT scan with contrast should be used as an adjunct to primary survey and aimed at detecting acute life-threatening injuries. This should be used where there is clinical evidence of bleeding or a high likelihood of vascular trauma. With the use of radiolucent scoops, patient transfer can be done within a short pace of time. CT scan is performed despite significant hypotension in advanced Trauma Centres. However, this should be done with caution in children under 2 years of age due to chances of exposure to high radiation.

\subsection{British Orthopaedic Association (BOA) Guidelines [20]}

The British Orthopaedic Association guidelines (BOAST 3) for acetabular and pelvic fracture management outline the need for CT scans for defining pelvic injury. However, they do recommend early CT scan if available in the emergency department unless it interferes with the initial resuscitation of the patient.

In case of genitourinary damage, cystography with or without CT and urethrography is recommended. Reduction of a native hip dislocation after trauma must be followed with a CT scan within 24 hrs to exclude bony entrapment and associated acetabular fracture.

In suspected cervical spine injuries (BOAST 2), a thin slice 2 - $3 \mathrm{~mm}$ helical CT scan from base of the skull to at least $\mathrm{T} 1$ with both coronal and sagittal reconstruction needs to be performed. Extending this scan to T4/5 can help to rule out upper thoracic spine injury at the same time. The remaining Lumbar spine may be visualised adequately by either plain AP and Lateral radiographs or by sagittal and coronal reformatting of helical CT scan of the chest, abdomen and Pelvis. Magnetic resonance imaging (MRI) is urgent in cases of spinal cord compression.

\section{CT Scans in Primary Survey}

Although several definitions exist to define critically injured patients and there are scoring systems to quantify them, major trauma usually refers to serious injuries to multiple body parts resulting in death or serious disabil- 
ity [21]. The importance of using CT scans in primary survey of polytraumatised patients is not new [22]. This involves refurbishing the CT suite to enable simultaneous resuscitation of patients whilst they have their scan. The decision to allow the trauma victim to undergo CT scan is taken by the trauma team leader in consultation with the surgical, orthopaedic and surgical teams. The CT scan however, should not delay or replace a thorough clinical examination. The information available on the haemodynamic status of the patients, the mechanism of injury and possible body parts involved determines if the patient can undergo a spiral CT scan. It is to be noted that MSCT exposes the patient to substantial radiation dose compared to conventional radiology [23].

If the patient is critical and needs urgent resuscitation in the emergency department, and CT scan is considered to be too time consuming, the diagnostic evaluation should be prioritised and undertaken in the emergency room. Conventional radiographs of chest and pelvis, cervical spine lateral view including C7 and FAST scan of the abdomen and pelvis is performed parallel to primary survey and initial resuscitation. The decision to undertake CT scan at this stage will be directed by his vital signs and results of the preliminary investigations.

In cases of less critical trauma, immediate CT scan is performed under continuous monitoring. Since resuscitation can be undertaken in the CT suite, hemodynamic instability should not be a contraindication to MSCT [2]. The body parts commonly scanned includes brain, cervical spine, chest, abdomen and pelvis including the hips. Any gross abnormality of the Iimbs (deformed arm or leg) can be incorporated in the CT at this stage on special request from the orthopaedic team. The findings are assessed immediately in a separate workstation by a trained radiologist and a preliminary report is produced in writing for the trauma team [24]. This allows the surgeon/ orthopaedic surgeon to plan surgical intervention. Furthermore, the CT image findings can be correlated to those found intraoperatively. This is of particular importance for centres practising damage control surgery as the decision to manage severe limb injuries can be based on exact nature of radiological images obtained from MSCT. A decision is taken to transfer the patient to theatre, intensive care unit or a major trauma centre soon after the patient comes out of the MSCT suite. Further imaging like radiographs of the extremities or angiograms can be planned at this stage. The radiologist performs further surveys of the CT findings and three-dimensional reconstructions are processed. Since this is more time consuming, relevant secondary CT findings are reported by telephone to the trauma team that is involved in the further management.

The patient is removed from the hard board but the cervical spine remains triple immobilised from his arrival to the emergency department until he/she is transferred to the operating table or the intensive therapy unit (ITU) bed. Furthermore, all patients are log rolled till the imaging excludes any unstable spinal injury. This ensures adequate immobilisation during transportation to different departments inside the hospital. The hard collar does not interfere with the procurement or quality of the CT images in any way. There is now growing evidence to support MSCT in primary survey of polytrauma patients with minimal delay [25,26].

\section{Conclusions}

Immediate CT scan can be a useful adjunct to primary survey in polytrauma patients. Most of the data to support this comes from central Europe $[7,18]$. However, it is endorsed in the guidelines published by BOA and RCR. The main limitation of MSCT at present is cost. It needs significant amount of investment to adequately locate and refurbish CT suites near emergency departments enabling simultaneous resuscitation of critically injured patients. It is not always financially viable to relocate existing CT suites or built new ones near the emergency departments especially in small district general hospitals in the UK. However, it is recommended that all newly built CT suites should be near the emergency departments ensuring quick transfer of patients with minimal movement. CT scans are increasingly used for initial assessment in some level 1-trauma centres in the UK [27]. The location of CT suites location is relative with many other factors like layout of the hospital, infection control, radiation protection, and safety protection. At present there is no published data on the cost effectiveness of relocating existing CT suites near emergency department. Financial benefits needs to be established before such guidelines are implemented.

A recent systematic review of whole body CT scans in multiple injured patients found no improvement in mortality compared to conventional imaging with supplemented CT scan during secondary survey [28]. The authors included four retrospective studies with varying sample sizes to conclude that although patients undergoing immediate CT spend significant less time in the emergency department, no further benefits can be proven. This result has to be taken with caution as one study had a large sample size compared to the other three and hence its results influenced the overall statistics performed. Furthermore there is a selection bias in each study as only the most severely injured patients [higher injury severity scores (ISS)] had MSCT compared to those who had conventional CT. The authors themselves acknowledged this limitation and advised future randomised controlled trails in patients with comparable injuries or ISS. At least on such trial is currently underway [29]. 
At present MSCT is recommended in tertiary centres dealing with regular polytrauma where necessary arrangements are in place to obtain imaging without compromising initial resuscitation. The trauma team leader however, takes this decision on clinical grounds.

\section{REFERENCES}

[1] G. Regel, P. Lobenhoffer, U. Lehmann, H. C. Pape, T. Pohlemann and H. Tscherne, "Results of the Treatment of Polytraumatized Patients. A Comparative Analysis of 3.406 Cases between 1972 and 1991,” Unfallchirurg, Vol. 96, No. 7, 1993, pp. 350-362.

[2] M. H. Hessmann, A. Hofmann, K. F. Kreitner, C. Lott and P. M. Rommens, "The Benefit of Multislice CT in the Emergency Room Management of Polytraumatized Patients," Acta Chirurgica Belgica, Vol. 106, No. 5, 2006, pp. 500-507.

[3] E. J. Mahoney, W. L. Biffl, D. T. Harrington and W. G. Cioffi, "Isolated Brain Injury as Cause of Hypotension in the Blunt Trauma Patient,” Journal of Trauma, Vol. 55, No. 6, 2003, pp. 1065-1069.

doi:10.1097/01.TA.0000100381.89107.93

[4] American College of Surgeons, "Advanced Trauma Life Support (ATLS) Instructor Manual,” 8th Edition, American College of Surgeons, Chicago, 2008.

[5] M. Bishop, W. C. Shoemaker, S. Avakian, et al., "Evaluation of a Comprehensive Algorithm for Blunt and Penetrating Thoracic and Abdominal Trauma," The American Surgeon, Vol. 57, No. 12, 1991, pp. 737-746.

[6] J. P. Pryor and P. M. Reilly, "Initial Care of the Patient with Blunt Polytrauma," Clinical Orthopaedics and Related Research, No. 422, 2004, pp. 30-36. doi:10.1097/01.blo.0000131245.72370.8c

[7] M. H. Hessmann, A. Hofmann, K. F. Kreitner, C. Lott and P. M. Rommens, "The Benefit of Multislice Computed Tomography in the Emergency Room Management of Polytraumatized Patients," European Journal of Trauma, Vol. 31, No. 3, 2005, pp. 231-238. doi:10.1007/s00068-005-2051-7

[8] T. E. Wurmb, P. Frühwald, W. Hopfner, T. Keil, M. Kredel, J. Brederlau, N. Roewer and H. Kuhnigk, "WholeBody Multislice Computed Tomography as the First Line Diagnostic Tool in Patients with Multiple Injuries: The Focus on Time,” Journal of Trauma, Vol. 66, No. 3, 2009, pp. 658-665. doi:10.1097/TA.0b013e31817de3f4

[9] T. Albrecht, J. von Schlippenbach, P. F. Stahel, W. Ertel and K. J. Wolf, “The Role of Whole Body Spiral CT in the Primary Work-Up of Polytrauma Patients-Comparison with Conventional Radiography and Abdominal Sonography,” Rofo, Vol. 176, No. 8, 2004, pp. 1142-1150. doi:10.1055/s-2004-813259

[10] P. Hilbert, K. zur Nieden, G. O. Hofmann, I. Hoeller, R. Koch and R. Stuttmann, "New Aspects in the Emergency Room Management of Critically Injured Patients: A Multi-Slice CT-Oriented Care Algorithm,” Injury, Vol. 38, No. 5, 2007, pp. 552-558. doi:10.1016/j.injury.2006.12.023
[11] C. D. Becker and P. A. Poletti, “The Trauma Concept: The Role of MDCT in the Diagnosis and Management of Visceral Injuries,” European Radiology, Vol. 15, No. S4, 2005, pp. 105-109. doi:10.1007/s10406-005-0122-9

[12] K. G. Kanz, M. Körner, U. Linsenmaier, M. V. Kay, S. M. Huber-Wagner, U. Kreimeier, K. J. Pfeifer, M. Reiser and W. Mutschler, "Priority-Oriented Shock Trauma Room Management with the Integration of Multiple-View Spiral Computed Tomography,” Der Unfallchirurg, Vol. 107, No. 10, 2004, pp. 937-944. doi:10.1007/s00113-004-0845-4

[13] M. Rieger, H. Sparr, R. Esterhammer, C. Fink, R. Bale, B. Czermak and W. Jaschke, "Modern CT Diagnosis of Acute Thoracic and Abdominal Trauma," Anaesthesist, Vol. 51, No. 10, 2002, pp. 835-842. doi:10.1007/s00101-002-0369-7

[14] T. Ptak, J. T. Rhea and R. A. Novelline, "Experience with a Continous, Single-Pass Whole-Body Multidetector CT Protocol for Trauma: The Three-Minute Multiple Trauma CT Scan,” Emergency Radiology, Vol. 8, No. 5, 2001, pp. 250-256. doi:10.1007/PL00011915

[15] D. Trunkey, "Initial Treatment of Patients with Extensive Trauma," The New England Journal of Medicine, Vol. 324, 1991, pp. 1259-1263. doi:10.1056/NEJM199105023241806

[16] T. P. Saltzherr, P. H. Fung Kon Jin, F. C. Bakker, et al., "An Evaluation of a Shockroom Located CT Scanner: A Randomized Study of Early Assessment by CT Scanning in Trauma Patients in the Bi-Located Trauma Center North-West Netherlands (REACT Trial),” BMC Emergency Medicine, Vol. 8, 2008, p. 10. doi:10.1186/1471-227X-8-10

[17] The Royal College of Radiologists, "Standards of Practice and Guidance for Trauma Radiology in Severely Injured Patients,” The Royal College of Radiologists, London, 2011.

[18] S. Huber-Wagner, R. Lefering, L. M. Qvick, M. Körner, M. V. Kay, K. J. Pfeifer, M. Reiser, W. Mutschler and K. G. Kanz, "Working Group on Polytrauma of the German Trauma Society. Effect of Whole-Body CT during Trauma Resuscitation on Survival: A Retrospective, Multicentre Study," Lancet, Vol. 373, No. 9673, 2009, pp. 1455-1456.

[19] NHS Clinical Advisory Group, "Regional Networks for Major Trauma.”

http://www.excellence.eastmidlands.nhs.uk/welcome/imp rov-ing-care/emergency-urgent-care/major-trauma/nhs-cli nical-advisory-group/

[20] British Orthopaedic Association, "Standards for Trauma (BOAST). Spinal Clearance in the Trauma Patient (BOAST 2) and Pelvic and Acetabular Fracture Managemnt (BOAST 3)," BOA, London, 2008.

[21] NHS Choices. http://www.nhs.uk/NHSEngland/AboutNHSservices/Eme rgencyandurgentcareservices /Pages/Majortraumaservices. Aspx

[22] B. Leidner, M. Adiels, P. Aspelin, P. Gullstrand and S. Wallén, "Standardized CT Examination of the Multitraumatized Patient,” European Radiology, Vol. 8, No. 9, 1998, 
pp. 1630-1638. doi:10.1007/s003300050601

[23] C. M. Hui, J. H. MacGregor, H. C. Tien and J. B. Kortbeek, "Radiation Dose from Initial Trauma Assessment and Resuscitation: Review of the Literature," Canadian Journal of Surgery, Vol. 52, No. 2, 2009, pp. 147-152.

[24] U. Linsenmaier, M. Krötz, H. Häuser, C. Rock, J. Rieger, K. Bohndorf, K. J. Pfeifer and M. Reiser, "Whole-Body Computed Tomography in Polytrauma: Techniques and Management,” European Radiology, Vol. 12, No. 7, 2002, pp. 1728-1740. doi:10.1007/s00330-001-1225-X

[25] P. Weninger, W. Mauritz, P. Fridrich, R. Spitaler, M. Figl, B. Kern and H. Hertz, "Emergency Room Management of Patients with Blunt Major Trauma: Evaluation of the Multislice Computed Tomography Protocol Exemplified by an Urban Trauma Centre,” Journal of Trauma, Vol. 62, No. 3, 2007, pp. 584-591. doi:10.1097/01.ta.0000221797.46249.ee

[26] R. Rossaint, B. Bouillon, V. Cerny, T. J. Coats, J. Duranteau, E. Fernández-Mondéjar, B. J. Hunt, R. Komadina, G. Nardi, E. Neugebauer, Y. Ozier, L. Riddez, A. Schultz, P. F. Stahel, J. L. Vincent and D. R. Spahn, "Task Force for Advanced Bleeding Care in Trauma. Management of
Bleeding Following Major Trauma: An Updated European Guideline,” Critical Care, Vol. 14, No. 2, 2010, p. R52. doi:10.1186/cc8943

[27] J. Gallagher, “Trauma Care: CT Scanner Is 'merged' with A\&E in Hospital First,” BBC News Health, 2012. http://www.bbc.co.uk/news/health-20174883.

[28] J. C. Sierink, T. P. Saltzherr, J. B. Reitsma, O. M. Van Delden, J. S. Luitse and J. C. Goslings, "Systematic Review and Meta-Analysis of Immediate Total-Body Computed Tomography Compared with Selective Radiological Imaging of Injured Patients,” British Journal of Surgery, Vol. 99, No. S1, 2012, pp. 52-58. doi:10.1002/bjs.7760

[29] J. C. Sierink, T. P. Saltzherr, L. F. Beenen, J. S. Luitse, M. W. Hollmann, J. B. Reitsma, M. J. Edwards, J. Hohmann, B. J. Beuker, P. Patka, J. W. Suliburk, M. G. Dijkgraaf and J. C. Goslings, (REACT-2 Study Group), “A Multicenter, Randomized Controlled Trial of Immediate TotalBody CT Scanning in Trauma Patients (REACT-2)," BMC Emergency Medicine, Vol. 12, 2012, p. 4. doi:10.1186/1471-227X-12-4 